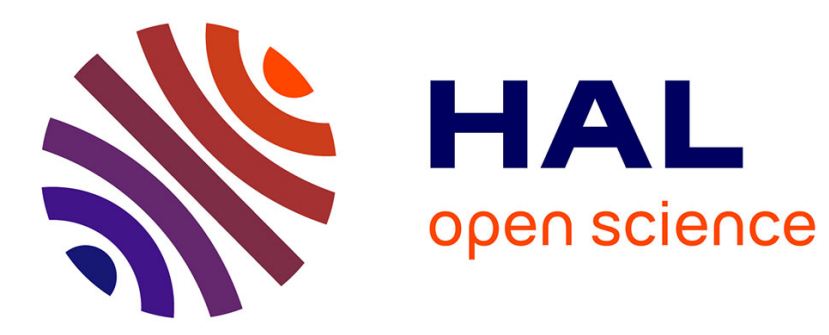

\title{
Design of a 3D Navigation Technique Supporting VR Interaction
}

\author{
Pierre Boudoin, Samir Otmane, Malik Mallem
}

\section{To cite this version:}

Pierre Boudoin, Samir Otmane, Malik Mallem. Design of a 3D Navigation Technique Supporting VR Interaction. First Mediterranean Conference on Intelligent Systems and Automation (CISA'08), Jun 2008, Annaba, Algeria. pp.149-153, 10.1063/1.2952967 . hal-00339450

\section{HAL Id: hal-00339450 https://hal.science/hal-00339450}

Submitted on 23 Jul 2009

HAL is a multi-disciplinary open access archive for the deposit and dissemination of scientific research documents, whether they are published or not. The documents may come from teaching and research institutions in France or abroad, or from public or private research centers.
L'archive ouverte pluridisciplinaire HAL, est destinée au dépôt et à la diffusion de documents scientifiques de niveau recherche, publiés ou non, émanant des établissements d'enseignement et de recherche français ou étrangers, des laboratoires publics ou privés. 


\title{
Design of a 3D Navigation Technique Supporting VR Interaction
}

\author{
Pierre Boudoin* \\ Samir Otmane* \\ Malik Mallem* \\ *Informatics, Integrative Biology and Complex Systems, \\ 40 Rue de Pelvoux, 91020 Evry Cedex, France \\ (e-mail: pierre.boudoin@ibisc.fr, samir.otmane@ibisc.fr, malik.mallem@ibisc.fr).
}

\begin{abstract}
Multimodality is a powerful paradigm to increase the realness and the easiness of the interaction in Virtual Environments (VEs). In particular, the search for new metaphors and techniques for $3 \mathrm{D}$ interaction adapted to the navigation task is an important stage for the realization of future 3D interaction systems that support multimodality, in order to increase efficiency and usability.

In this paper we propose a new multimodal 3D interaction model called Fly Over. This model is especially devoted to the navigation task. We present a qualitative comparison between Fly Over and a classical navigation technique called gaze-directed steering. The results from preliminary evaluation on the IBISC semi-immersive Virtual Reality/Augmented Realty EVR@ platform show that Fly Over is a user friendly and efficient navigation technique.
\end{abstract}

Keywords: 3D Interaction Techniques, Multimodality, Navigation Task, Virtual Environment

\section{INTRODUCTION}

$\mathrm{M}$ ultimodality is a powerful paradigm to increase the realness and the easiness of the interaction in Virtual Environments (VEs). In particular, the search for new metaphors and techniques for 3D interaction adapted to the navigation task is an important stage for the realization of future $3 \mathrm{D}$ interaction systems that support multimodality, in order to increase efficiency and usability.

In this paper we propose a new multimodal $3 \mathrm{D}$ interaction model called Fly Over. This model is especially devoted to the navigation task. We present a qualitative comparison between Fly Over and a classical navigation technique called gaze-directed steering. The results from preliminary evaluation on the IBISC semi-immersive Virtual Reality/Augmented Realty EVR@ platform show that Fly Over is a user friendly and efficient navigation technique.

\section{RELATED WORK}

The navigation task is probably the most utilized task in VEs [Bowman and al., 2005]. The aim of this task is to give the user the feeling he is moving naturally and easily in a VE, whereas avoiding sickness feelings.

A lot of navigation techniques have already been developed. However, they are highly dependent from hardware interface. Indeed:

- Gaze-directed steering technique [Mine, 1995] needs user's head tracking;

- Pointing technique [Bowman and al., 1997] needs user's hand tracking;

- Map-based travel technique [Bowman and al., 1998] needs a 2D display and a pointer;
- Grabbing the air technique [Mapes, and al., 1995] needs pinch-gloves.

These techniques are efficient for an isolated navigation task. But if we consider a global action in a VE (including navigating, selecting or manipulating objects), different devices may be needed and switches between tasks and devices may be difficult to handle for the user.

Hence, multimodal framework is needed. We have studied different multimodal frameworks, especially devoted to devices management. Two drew our attention:

The Sylvia Irawati's team [Irawati and al., 2006] proposed a complete framework. The most interesting part is their using of objects ontology to make the interaction more natural and user-friendly. Moreover the object ontology they've proposed supports constraints definitions. It could be interesting to be inspired by some parts of their framework.

The Ed Kaiser's team [Kaiser and al., 2003] worked on mutual disambiguation. Their work testified an interesting approach to manage multimodality with the use of what they called Multimodal Integrator. The aim of this integrator is to find the best multimodal interpretation with the preliminary rated inputs. The principle is to unify inputs data in:

Amalgamating redundant or complementary data via a logical test set;

- Taking care about the spatiotemporal aspect of data;

- Taking care about data's hierarchy.

\section{THE FLY OVER MODEL}

We propose a new 3D interaction model - called Fly Over based on the following four constraints:

- To be compatible with all common 2D, 3D or 6D devices (mouse, hand/head/finger tracking, force feedback) that could 
return a $2 \mathrm{D} / 3 \mathrm{D}$ position/orientation of the user or an object he manipulates;

- To maintain the same logic of use for all devices, even if the employed technologies are very different from each other; -to be natural;

-to be associated to a short training duration.

\subsection{Generic model specification}

The Fly Over model may be depicted as a blob, which is composed of interaction areas, modeled as concentric spheres (see figure 1). Each interaction area is a subspace of the whole task space. For example, figure 1 depicts a 6D task space in VE parted into two 3D subspaces.

The action of the user on VE may be summarized, at each time: $t$, by a vector $\mathrm{P}$ (6 components for a $6 \mathrm{D}$ task). Each interaction area $\mathrm{Zi}$ is associated to specific sub-vector $\mathrm{Pi}$ of $\mathrm{P}$.

As stated by constraint 1, the user's action on the device he utilizes may control a pointer (modeled as a tiny sphere in figure 1) in VE. The presence of the pointer into area $\mathrm{Zi}$ is translated into a modification of the sub-vector Pi.

The crossing from one interaction area to another allows the user to modify consecutively all the components of vector $P$.

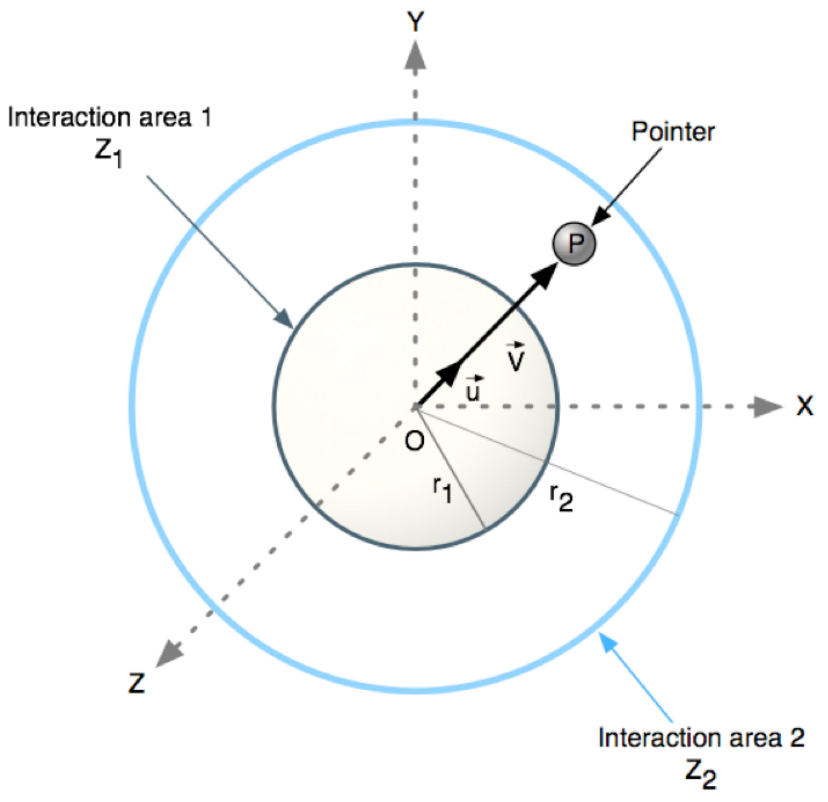

Fig. 1. Fly Over blob with two 3D interaction areas Z1 and $\mathrm{Z2}$ designed for a $6 \mathrm{D}$ task in VE.

In order to fulfill the last two conditions (natural technique and fast learning), we decided to use the simple virtual hand technique to handle the virtual pointer move in VE.

\subsection{Generic model parameters}

We can access to five parameters via the generic model. These parameters are computed with the only knowledge of position of the effector controlled by the user.
-First parameter: Position vector of pointer $\vec{V}=\overrightarrow{O P}$

This parameter indicates the position of the effector relative to the blob. It may be interpreted as a position, a translation vector, a direction and so defining an orientation

-Second parameter: presence in the interaction area 1

$$
\left\{\begin{array}{lll}
P \in Z_{1} & \text { Si } & \overrightarrow{\|V\|}<r_{1} \\
P \notin Z_{1} & \text { Si } & \overrightarrow{\|V\|}>r_{1}
\end{array}\right.
$$

-Third parameter: Presence in the interaction area 2

$$
\left\{\begin{array}{lll}
P \in Z_{2} & \text { Si } & r_{1}<\overrightarrow{\|V\|}<r_{2} \\
P \notin Z_{2} & \text { Si } & \overrightarrow{\|V\|}>r_{2} \text { ou } \overrightarrow{\|V\|}<r_{1}
\end{array}\right.
$$

-Fourth parameter: Global intensity

$$
I=\overrightarrow{\|V\|}
$$

-Fifth parameter: Intensity in the 3D interaction area 2

$$
I_{2}=\overrightarrow{\|V\|}-r_{1}
$$

\subsection{Fly Over for navigation task: Fly Over $-\mathrm{N}$}

The generic Fly Over model was firstly designed for navigation tasks. Indeed, we have previously noticed that:

- Managing simultaneously translation and rotation in VE on a (semi-)immersive VR platform may cause nausea for the user;

- The users naturally choose their orientation in order to have the aimed object in front of them, and then translate to the object.

So, these observations were compatible with the fact that, within the Fly Over model, it is possible to decouple the 6D navigation task into two 3D subspaces: a subspace dedicated to the position of the user in VE and another one dedicated to the orientation of the user in VE. This leads to the Fly Over $\mathrm{N}$ model depicted in figure 2 .

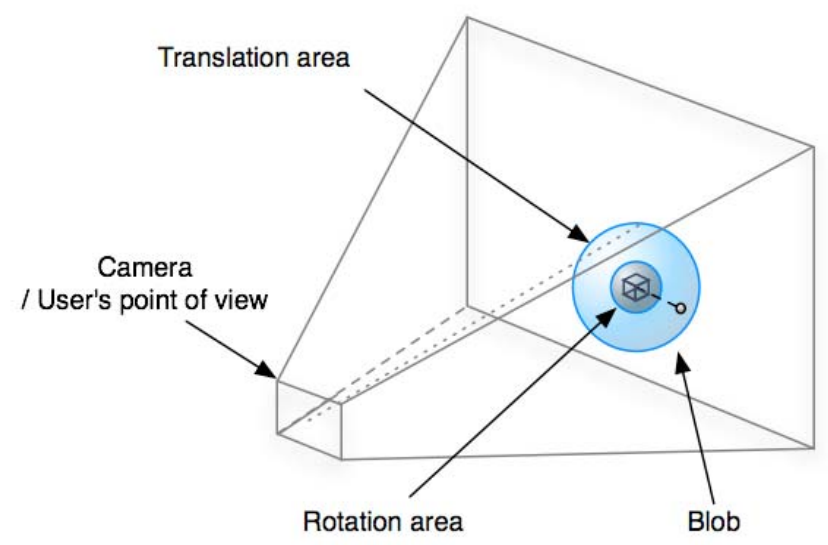

Fig. 2. Fly Over - $\mathrm{N}$ model. The 6D navigation space is parted into two 3D subspaces: $\mathrm{Z1}$ dedicated to rotations and $\mathrm{Z2}$ dedicated to translations. 
The position of the pointer in the rotation area leads to a rotation of the user in VE with magnitude I whereas the position of the pointer in the translation area leads to a translation of the user in VE with magnitude I2.

The generic algorithm described in section 3.2 becomes:

$$
\begin{aligned}
& \text { START: } \\
& \text { IF }\left(\overrightarrow{\|V\|}<r_{1}\right) \text { THEN } \\
& \text { Orientation }(\vec{V}) ; \\
& \text { ELSE } \\
& \text { IF }\left(r_{1}<\overrightarrow{\|V\|}<r_{2}\right) \text { THEN } \\
& \quad / / \text { Computing the translation vector ; } \\
& \quad \vec{T}=\operatorname{Normalize}(\vec{V}) * I_{2} ; \\
& \quad \text { Translation }(\vec{T}) ; \\
& \text { END }
\end{aligned}
$$

Where:

- Orientation $(\vec{V})$ : execute the necessary rotations in order to direct the camera in the direction given by $\vec{V}$.

- Translation $(\vec{V})$ : execute the translation given $\vec{V}$.

- Normalize $(\vec{V})$ : normalize the vector $\vec{V}$ in order to transform it in unit vector.3.3. Visual assistances going with Fly Over - N

In order to help users when they navigate in VE, visual assistances are displayed. The blob is displayed in a translucent way and is placed in front of the virtual camera, which represents the point of view of user (see figure 2).

The first interaction area will be blue tinted and the second won't be tinted and vice versa, depending on the presence of the pointer into these areas (see figure 3). A wire-frame cube is displayed to symbolize the effective orientation of the user in

VE.
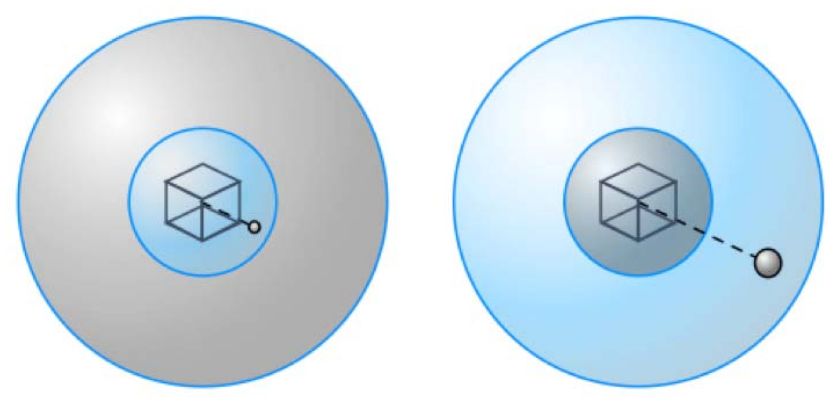

Fig. 3. Visual assistances in the Fly Over blob.

\section{HARDWARE AND SOFTWARE IMPLEMENTATION}

Our experiments has been performed on the IBISC Lab. semi-immersive multimodal EVR@ platform (see figure 4), which permits to follow the gestures of the user's hand and finger positions (wireless Flystick 1 coupled to two ARTTrack1 infrared cameras, wireless 5DT data gloves Ultra
14) and has a 6D force feedback device (SPIDAR-G [Sato, 2006]).

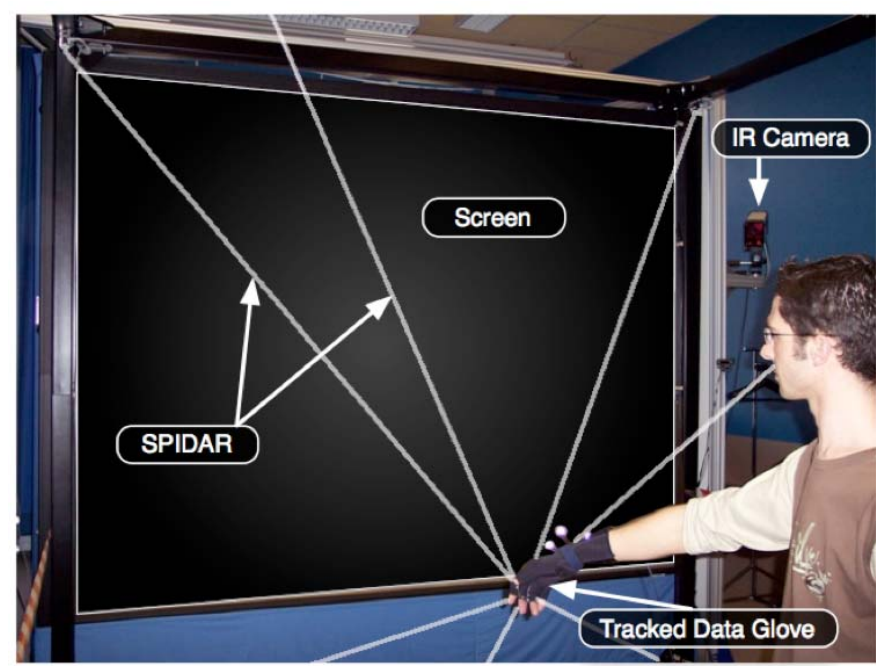

Fig. 4. The EVR@ platform. We can see Data Gloves, Optical Tracking System and SPIDAR in use.

Each device is associated with a specific server. We utilized the VRPN library [Taylor II and al., 2001] to implement the gathering of all our data from the different servers and Virtools ${ }^{\mathrm{TM}}$ to make the interactive virtual environments needed in our experiments.

\section{PRELIMINARY EVALUATION}

In order to realize a preliminary evaluation, we compared Fly Over - N (FO-N) to gaze-directed steering (GDS). A total of 12 young students, including 10 males and 2 females participated to the experiment. 2 of them considered themselves as experts in using VE systems whereas 4 considered themselves as intermediate and 6 as beginners. However, none of them have already utilized FO-N nor GDS.

VE was the representation of a part of the IBISC Lab. For FO-N, the device used to navigate was a Flystick. Figure 5 shows the experimental setting.

The participants were asked to follow 3 times as precisely as possible a trajectory in VE depicted with a thin red line, going from point A to point B (see figure 6). Duration of the experiment was not considered.

The target trajectory was built to be sinuous. The main question was: is it easier to follow the target trajectory with the help of FO-N than with GDS?

Data showed that the use of FO-N gives smoother trajectories than GDS (figure 7). However, there exists a bias for FO-N: users are doing trajectories that are near from the target trajectory but not centered on it.

Participants were given qualitative questionnaires after the experiment: Q1-Did you find easy to learn the FO-N/GDS?, Q2-Did you found easy to navigate with FO-N/GDS?, Q3Did you found easy to follow the target trajectory?, Q4-Did 
you feel sickness? The possible answers were Agree, Neutral and Disagree.

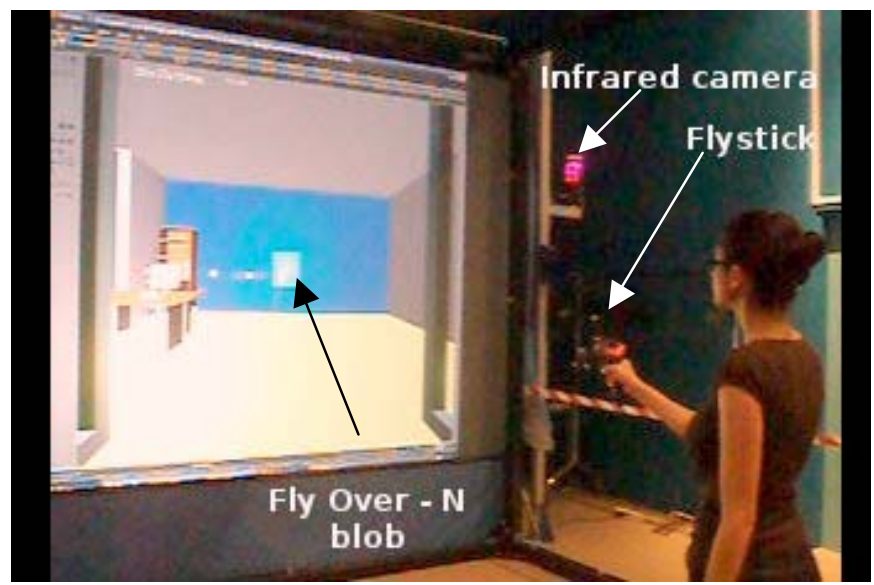

Fig. 5. Experimental setting with the use of FO-N on the IBISC semi-immersive EVR @ platform. Users navigate by moving a Flystick in their hand, which position is computed by two infrared cameras.

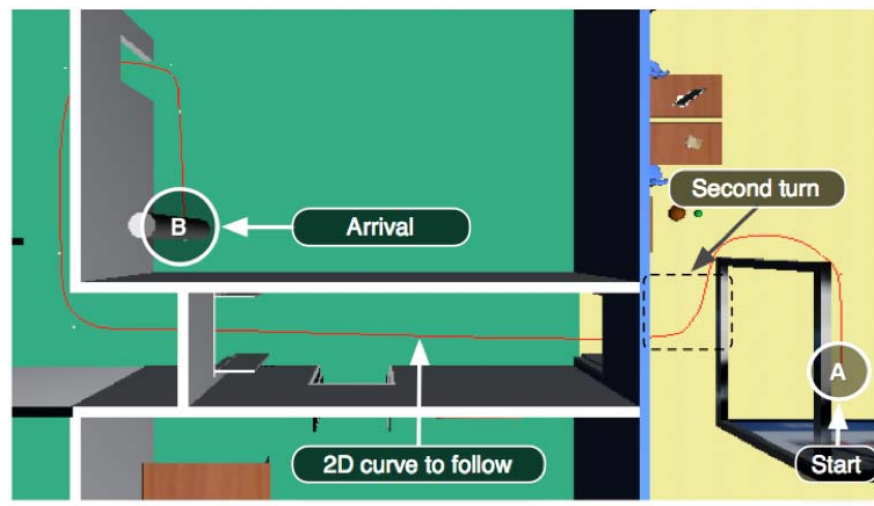

Fig. 6. Course to follow with target trajectory.

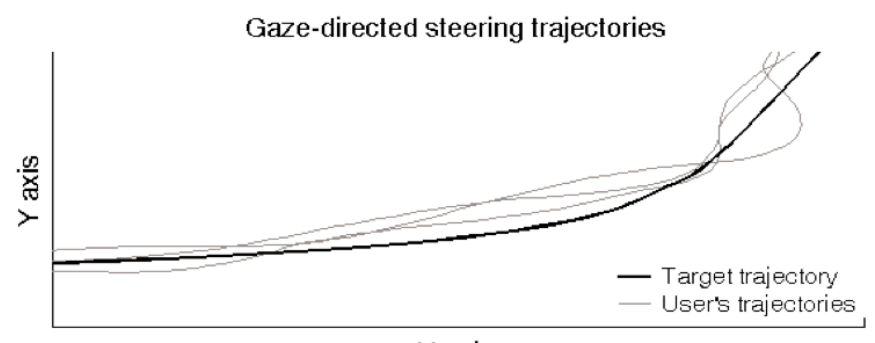

$X$ axis

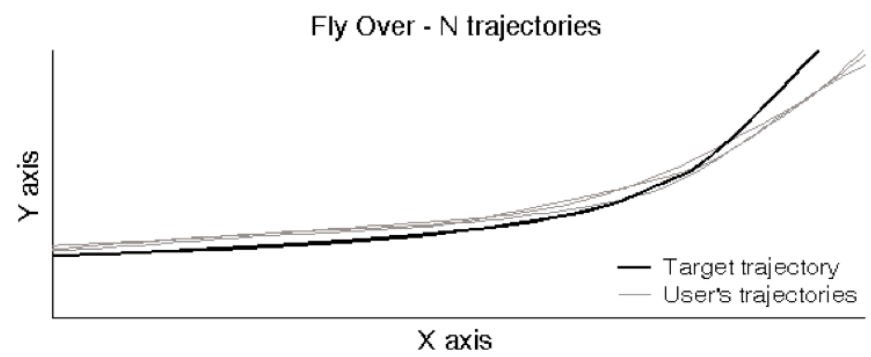

Fig. 7. Trajectories comparison for one user in the second turn of GDS and FO- N techniques.

The results show that FO-N was preferred and seems to be easier and more usable than GDS. Indeed, for Q1, we got a
9A-3N-0D for FO-N and a 8A-3N-1D for GDS. For Q2, we got a $8 \mathrm{~A}-3 \mathrm{~N}-0 \mathrm{D}$ for FO-N whereas GDS obtained a $2 \mathrm{~A}-1 \mathrm{~N}$ 9D which tend to show that users were not easy with GDSI. For Q3, we got a 5A-4N-3D for FO-N whereas GDS obtained a 3A-3N-6D. Finally, for Q3 we obtained a 0A-1N$11 \mathrm{D}$ for FO-N whereas GDS got a $3 \mathrm{~A}-1 \mathrm{~N}-8 \mathrm{D}$, which means that all the users felt comfortable with FO-N whereas a minority of them felt sickness with GDS.

\section{CONCLUSION AND PROSPECTS}

In this paper, we propose a new multimodal $3 \mathrm{D}$ interaction model, called Fly Over. This model is generic and is based on two main ideas. First, all basic 3D interaction tasks may be turned into a simple pointing task. Second, the $6 \mathrm{D}$ space of the user (3D position and 3D orientation) may be seen as a set of hyperspaces in which a separate pointing task may be applied. Due to these ideas, Fly Over may be utilized the same way with various $2 \mathrm{D}, 3 \mathrm{D}$ or $6 \mathrm{D}$ devices.

The generic model has been applied to a $2 \mathrm{D}$ navigation task and has been compared to the gaze-steering technique. Preliminary qualitative results obtained on the IBISC semiimmersive Virtual Reality EVR@ platform shows that Fly Over generates smoother trajectories and is well accepted by the users.

Ongoing work is concerning the evaluation of Fly Over for a real 3D navigation task in submarine environments (French ANR Digital Ocean project). We predict that the splitting between 3D orientation and 3D position will have a benefic effect on the easiness of the 3D navigation task.

As the Fly Over model is generic, future work will be held on the application on manipulation and system command tasks. Our goal will be to show if our technique leads to a continuity feeling between tasks when switching from a device to another, and if the total training time is lessen, as we suppose to be.

\section{ACKNOWLEDGMENTS}

This work is supported by the VarSCW (Virtual and augmented reality Supported Collaborative Work) project. We wish to thank "Le Conseil Général De 1' Essonne", "Le C.N.R.S." and the IBISC laboratory for funding this project

\section{REFERENCES}

[Bowman and al., 1997] D.A. Bowman, D. Koller, L. Hodges (1997). Travel in Immersive Virtual Environments: an Evaluation of Viewpoint Motion Control Techniques. In: Proceedings of the Virtual Reality Annual International Symposium, pp. 45-52.

[Bowman and al., 1998] D.A. Bowman, J. Wineman, L. Hodges, D. Allison (1998). Designing Animal Habitats Within an Immersive VE. IEEE Computer Graphics \& Applications, 18(5), pp. 9-13.

[Bowman and al., 2005] D.A. Bowman, E. Kruijff, J.J. LaViola, I. Poupyrev (2005). 3D user interfaces: Theory and Practice, pp. 1-26, 87-287. Addison- Wesley. 
[Irawati and al., 2006] S. Irawati, D. Calderòn, H. Ko (2006). Spatial Ontology for Semantic Integration in 3D Multimodal Interaction Framework. In: VRCIA, pp. 129135. Hong Kong.

[Kaiser and al., 2003] $\quad$ E. Kaiser , A. Olwal, D. McGee , H. Benko, A. Corradini, X. Li, P. Cohen and S. Feiner S (2003). Mutual Disambiguation of 3D Multimodal Interaction. In: ICMI-PUI' 03 .

[Mapes and al., 1995] $\quad$ D. Mapes, J. Moshell (2005). A Two-Handed Interface for Object Manipulation in Virtual Environments. In: Presence: Teleoperators and Virtual Environments, 4(4), pp. 403-416.

[Mine, 1995] M. Mine (1995). Virtual Environment Interaction Techniques (Technical Report TR95-018). UNC Chapel Hill CS Dept.

[Sato, 2006] M. Sato (2006). A String-based Haptic Interface "SPIDAR. In: ISUVR2006, 191.

[Taylor II and al., 2001] R.M. Taylor II, T.C. Hudson, A. Seeger, H. Weber, J. Juliano, A.T. Helser (2001). VRPN: A Device-Independent, Network-Transparent VR Peripheral System. In: ACM Symposium on Virtual Reality Software and Technology, pp. 56-61. 